\title{
Supernova remnants and the origin of cosmic rays
}

\author{
Jacco Vink \\ Astronomical Institute Anton Pannekoek \& GRAPPA \\ Universiteit van Amsterdam \\ Postbus 94249 \\ 1090 GE Amsterdam, The Netherlands \\ email: j.vink@uva.nl
}

\begin{abstract}
Supernova remnants have long been considered to be the dominant sources of Galactic cosmic rays. For a long time the prime evidence consisted of radio synchrotron radiation from supernova remnants, indicating the presence of electrons with energies of several GeV. However, in order to explain the cosmic ray energy density and spectrum in the Galaxy supernova remnant should use $10 \%$ of the explosion energy to accelerate particles, and about $99 \%$ of the accelerated particles should be protons and other atomic nuclei.

Over the last decade a lot of progress has been made in providing evidence that supernova remnant can accelerate protons to very high energies. The evidence consists of, among others, $\mathrm{X}$-ray synchrotron radiation from narrow regions close to supernova remnant shock fronts, indicating the presence of 10-100 $\mathrm{TeV}$ electrons, and providing evidence for amplified magnetic fields, gamma-ray emission from both young and mature supernova remnants. The high magnetic fields indicate that the condition for accelerating protons to $>10^{15} \mathrm{eV}$ are there, whereas the gamma-ray emission from some mature remnants indicate that protons have been accelerated.
\end{abstract}

Keywords. acceleration of particles, magnetic fields, radiation mechanisms: nonthermal, shock waves, (stars:) supernovae: general, (ISM:) cosmic rays,(ISM:) supernova remnants

\section{Introduction}

The energy density of cosmic rays in the Galaxy is estimated to be $1-3 \mathrm{eV} \mathrm{cm}^{-3}$ (e.g. Webber 1998), which is similar to the internal gas density and magnetic field energy density in the interstellar medium (ISM). The idea that the bulk of these cosmic rays are associated with supernovae was first made by Baade \& Zwicky (1934b), who also for the first time made a clear distinction between "super novae" and "common novae" (Baade \& Zwicky 1934a).

The discovery of radio emission from supernova remnants (SNRs) and its identification as synchrotron radiation in the 1950ies (e.g. Ginzburg 1959) provided the first link between supernovae and particle acceleration. Of course, synchrotron radiation is primarily associated with relativistic electrons and positrons, whereas $99 \%$ of the cosmic rays observed on Earth consists of electrons. At the time it was not quite clear whether the acceleration occurred during the supernova explosion or in the SNR shells (Ginzburg \& Syrovatskii 1964). The radio flux decline of the bright SNR Cassiopeia A, and the explanation by Shklovsky that this was caused by the adiabatic cooling of the relativistic electrons even suggested that electrons were no longer actively accelerated anymore (Shklovsky 1968).

The idea that the shocks at the outer boundaries of SNRs were the likely sites of cosmic ray acceleration became more prominent due to development of the theory of first order Fermi acceleration, also know as diffusive shock acceleration (DSA) (Axford 
et al. 1977; Krymskii 1977; Blandford \& Ostriker 1978; Bell 1978). According to this theory particles gain momentum by repeatedly crossing the shock front as a result of particle diffusion. Each shock crossing leads to a fractional increase in momentum of order $\Delta p / p \approx \Delta v / c$, with $\Delta v$ the difference in plasma velocity between both sides of the shock and $c$ the particle velocity. The particle distribution becomes a power law, because the advection of plasma away from the shock in the downstream region (i.e. in the shock heated plasma), transports a fraction of $\sim v /(c \chi)$ (with $\chi$ the shock compression ratio) away from the shock region, preventing these particles from recrossing the shock again. The power law index of the energy spectrum, $q$, is for that reason a function of the compression ratio: $q=(\chi+2) /(\chi-1)$.

Although this theory made the case for shock acceleration in SNRs stronger, a number of problems remained in connecting the bulk of the cosmic rays with SNRs. For SNRs to be the origin of cosmic rays two criteria have to be met: 1) SNRs should be able to provide sufficient power to explain the cosmic ray energy density in the galaxy of $1 \mathrm{eV} / \mathrm{cm}^{-3}$, which requires that about $10 \%$ of the supernova explosion energy should be used to accelerate particles (Ginzburg \& Syrovatskii 1964); 2) SNRs should be able to accelerate protons at least up to $3 \times 10^{15} \mathrm{eV}$, which corresponds to a break in the observed cosmic ray spectrum, and probably marks the maximum energy that protons can be accelerated to in Galactic sources.

Applying shock acceleration theory to SNRs shows that it is difficult to fulfil the second criterion. The maximum energy to which particles can be accelerated by DSA depends on the time available for particle acceleration, the shock speed, and on the diffusion coefficient as a function of particle energy. The scattering causing the diffusion caused by turbulent magnetic fields. For the diffusion coefficient one often assumes that it is $D=\frac{1}{3} \eta c r_{\mathrm{g}}$, with $r_{\mathrm{g}}=E / e B$ the gyro radius radius of the particle, and $\eta$ a parameter indicating how much the mean free path is larger than the gyro-radius. The smallest diffusion coefficient, which gives the fastest acceleration, is thought to be $\eta=1$ (but see Reville \& Bell 2013). The case $\eta=1$ is referred to as "Bohm diffusion". In Lagage \& Cesarsky (1983) it was shown that under some general assumptions it seems unlikely that SNRs can really accelerate particles to beyond $3 \times 10^{15} \mathrm{eV}$. A more realistic estimate seemed to be $10^{14} \mathrm{eV}$, which even involved assuming the optimistic case that $\eta=1$, and that the magnetic field is that of the ISM $B \approx 3 \mu \mathrm{G}$.

As for the first criterion, for a long time the estimate of the total energy in cosmic rays in SNRs had to rely on the observed synchrotron luminosity. Translating this into an particle energy density has to rely on assumptions on the magnetic field strength and the ratio between accelerated electron and proton density. For the magnetic field strength one usually relied on equipartition arguments, whereas for the electron/proton ratio it was usually assumed that electrons account for about $1 \%$ of the particles, based on the electron/proton ratio in the cosmic rays observed on Earth.

Over the last decade our understanding of cosmic rays inside SNRs has greatly improved, thanks to new X-ray observatories, like Chandra, XMM-Newton, and Suzaku, and thanks to new gamma-ray observatories like ground-based Cherenkov telescopes that cover the gamma-rays above $\sim 0.1 \mathrm{TeV}$ and the Fermi and AGILE satellites which observe gamma-rays in the $\sim 0.1-10 \mathrm{GeV}$ range.

I will cover here these recent advances, but would like to point out that more extended reviews on high energy emission from SNRs are Reynolds (2008), Hinton \& Hofmann (2009), Vink (2012b), and Helder et al. (2012). In this volume there is overlap with the work presented by T. Brandt, S. Gabici, M. Lemoine-Goumard, on the gamma-ray emission, and with A. Decourchelle and M. Miceli on the X-ray emission. 


\section{X-ray synchrotron emission}

Although synchrotron radiation only inform us about the accelerated electrons (leptonic cosmic rays), the discovery of X-ray synchrotron emission from young SNRs gives us important information about particle acceleration in general. Moreover, X-ray telescopes like XMM-Newton, and in particular, Chandra have a spatial resolution that is orders of magnitude better than that of gamma-ray observatories.

$\mathrm{X}$-ray synchrotron radiation comes from the highest energy electrons. The characteristic frequency/photon energy of synchrotron radiation depends on electron energy as

$$
h \nu_{\mathrm{ch}}=13.9\left(\frac{B_{\perp}}{100 \mu \mathrm{G}}\right)\left(\frac{E}{100 \mathrm{TeV}}\right)^{2} \mathrm{keV} .
$$

This tells us immediately that synchrotron radiation above $1 \mathrm{keV}$ requires either high magnetic fields, or electrons with energies of $\sim 10^{13} \mathrm{eV}$ have to be present.

For that reason the first discovery of X-ray synchrotron radiation from the limbs of the shell-type SNR SN1006 (Koyama et al. 1995) was very important, as it indicated that electrons could be accelerated to energies much higher than was inferred before. Moreover, electrons with $\mathrm{TeV}$ energies loose their energy relatively quickly, with a typical loss time scale of

$$
\tau_{\mathrm{syn}}=\frac{E}{d E / d t}=12.5\left(\frac{E}{100 \mathrm{TeV}}\right)^{-1}\left(\frac{B_{\mathrm{eff}}}{100 \mu \mathrm{G}}\right)^{-2} \mathrm{yr} .
$$

So the presence of $\mathrm{TeV}$ electrons close to the shock front, also tell us that the acceleration is still taking place, or at least took place relatively recently. This clearly identifies the shock front of the SNR as a site of acceleration.

Finally, the time scale for acceleration to an energy $E$ by DSA is estimated to be

$$
\tau_{\text {acc }} \approx 1.83 \frac{D_{2}}{V_{\mathrm{s}}^{2}} \frac{3 \chi^{2}}{\chi-1}=124 \eta B_{-4}^{-1}\left(\frac{V_{\mathrm{s}}}{5000 \mathrm{~km} \mathrm{~s}^{-1}}\right)^{-2}\left(\frac{E}{100 \mathrm{TeV}}\right) \frac{\chi_{4}^{2}}{\chi_{4}-\frac{1}{4}} \mathrm{yr},
$$

with $B_{-4}$ the downstream magnetic field in units of $100 \mu \mathrm{G}$ and $\chi_{4}$ the overall compression ratio in units of 4 , and $V_{\mathrm{s}}$ the shock velocity. So in order to accelerate faster than the synchrotron loss time, for a $10 \mathrm{TeV}$ electron, one needs a factor of $\eta$ that cannot be too large compared to Bohm diffusion.

The maximum photon energy for synchrotron radiation can be calculated by making either the assumptions that the electron energy was limited by the time available to reach the maximum (Eq. 2.3), in which case the maximum electron energy must equal the maximum proton energy, or by assuming that the electron energy is limited by synchrotron losses. The latter can be calculated by setting $\tau_{\text {acc }}=\tau_{\text {syn }}$, which results in an equation that still depends on $B$. However, converting then electron energy to characteristic photon energy gives

$$
h \nu_{\max }=1.4 \eta^{-1}\left(\frac{\chi_{4}-\frac{1}{4}}{\chi_{4}^{2}}\right)\left(\frac{V_{s}}{5000 \mathrm{~km} \mathrm{~s}^{-1}}\right)^{2} \mathrm{keV},
$$

which does not depend on $B$ (Aharonian \& Atoyan 1999; Zirakashvili \& Aharonian 2007). If indeed the maximum electron energy is loss-limited the detection of X-ray synchrotron radiation indicates that $\eta \lesssim 10$.

The idea that for most young SNRs the synchrotron spectra are loss-limited is strengthened by the fact that the X-ray synchrotron emitting region is rather narrow (Helder et al. 2012, for an overview). In some cases, like Tycho's SNR (Fig. 1, Warren et al. 2005) and Cassiopeia A (Vink \& Laming 2003), the synchrotron rims are only a few arcsec in width, and can only be resolved by Chandra. The easiest explanation is that the width is 
determined by the loss-time scale: while the electrons are advected away from the shock they loose energy and after some time $\tau_{\text {syn }}$ they no longer emit X-rays. This would lead to the following expression for the rim width (Vink \& Laming 2003)

$$
l_{\mathrm{adv}}=\Delta v \tau_{\mathrm{syn}}=\left(V_{\mathrm{s}} / \chi\right) \tau_{\mathrm{syn}} .
$$

Alternatively one assume that the rim width corresponds to the typical electron diffusion length scale (e.g. Berezhko et al. 2003; Bamba et al. 2004),

$$
l_{\text {diff }} \approx D / V_{\mathrm{s}} .
$$

In fact the two approximations are both valid if the electrons are near their maximum energies, since then $\tau_{\text {syn }}=\tau_{\text {acc }}$, and $\tau_{\text {acc }} \approx D / V_{s}^{2}$, which can be easily combined to give Eq. 2.5. Under the assumption that $\tau_{\text {syn }}=\tau_{\text {acc }}$ one can even find an expression for the magnetic field which is independent of the observed photon energy and electron energy:

$$
B_{2} \approx 26\left(\frac{l_{\mathrm{adv}}}{1.0 \times 10^{18} \mathrm{~cm}}\right)^{-2 / 3} \eta^{1 / 3}\left(\chi_{4}-\frac{1}{4}\right)^{-1 / 3} \mu \mathrm{G},
$$

with $B_{2}$ the typical magnetic field strength in the shocked plasma.

The fact that using either Eq. 2.5 or Eq. 2.6 gives very similar magnetic field estimates (e.g. Ballet 2006), strengthens the case that the X-ray synchrotron emission is from loss-limited electron spectra. However, the low magnetic fields of some older X-ray synchrotron emitting SNRs may indicate the synchrotron spectrum is close to age-limited, or otherwise evolutionary effects should be taken into account. An example is RCW 86 for which $B_{2} \approx 20-30 \mu \mathrm{G}$. Also for the youngest known Galactic SNR, G1.9+0.3, it is debatable whether the maximum electron energy is limited by the age of the SNR ( 100 yr Carlton et al. 2011), or by radiative losses.

One of the surprising results that came out of the estimation of $B_{2}$ based on Eq. 2.7 or similar equations is that the magnetic field in young SNRs is much higher than anticipated, with typically $20 \mu \mathrm{G}<B_{2}<600 \mu \mathrm{G}$ (Vink \& Laming 2003; Berezhko et al. 2003; Bamba et al. 2004; Ballet 2006). Moreover, there is a clear correlation with local circumstellar density and shock speed, which indicates that $B_{2}^{2} \propto \rho_{0} V_{s}^{\alpha}$, with $\alpha$ in the range 2-3 (Völk et al. 2005; Vink 2008; Helder et al. 2012). Note that all young SNRs have velocities in a narrow range (3000-6000 $\left.\mathrm{km} \mathrm{s}^{-1}\right)$, making $\alpha$ poorly constrained, whereas the dynamical contrast in $\rho$ is much larger.

The magnetic fields are clearly larger than what can be expected from a compressed ISM magnetic field, which alleviates the problem discussed by Lagage \& Cesarsky (1983), namely that the magnetic fields are not high enough to have protons accelerated to $3 \times 10^{15} \mathrm{eV}$. The strong magnetic fields also support the idea that somehow magnetic field amplification near the shock is taking place, which in itself may be caused by the precursor resulting from efficient cosmic ray acceleration (e.g. Bell 2004; Drury \& Downes 2012).

That magnetic field amplification may be very efficient is indicated by the narrow Xray synchrotron filaments in the inside of Cas A, peaking in the west of the SNR (Helder \& Vink 2008; Uchiyama \& Aharonian 2008). Its location strongly suggest that these filaments are connected to the reverse shock of the SNR. Proper motion measurements in that region indicate that in our frame the reverse shock is almost at a stand still (Vink et al. 1998; Delaney \& Rudnick 2003), indicating that the shock speed is almost equal to the free expansion velocity of the ejecta $\sim 7000 \mathrm{~km} \mathrm{~s}^{-1}$. What is surprising about acceleration and magnetic field amplification at the reverse shock is that the unshocked ejecta are likely to have a low magnetic field, because of the large expansion of the frozen 

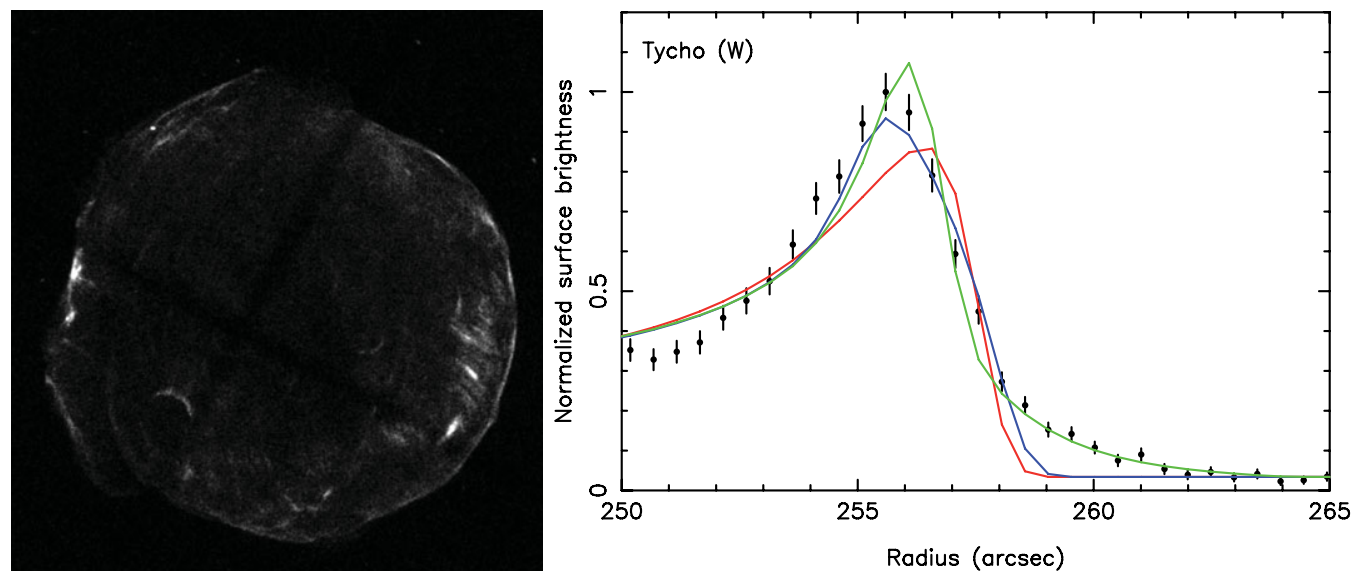

Figure 1. Left: Chandra X-ray image (2009) of Tycho SNR in the 4.6-6.1 keV band, which is dominated by X-ray synchrotron radiation. Note the narrow rim, in particular on the Western side, and the stripe-like pattern more inside the periphery in the West (Eriksen et al. 2011). Right: Emission profile from a $30^{\circ}$ segment of the Western shock region in the 4.3-5.2 keV band. The blue line shows emission from a uniformly emitting shell with thickness $l$. The red and green lines show models in which the emissivity falls off behind the shock as $\exp \left(-\left(r_{0}-r\right) / l\right)$, but the green line has an additional precursor component. The models take into account spherical projection and instrumental resolution (taken from Vink 2012a).

in magnetic field. Nevertheless, the width of the filaments indicate magnetic fields of the order of $100-500 \mu \mathrm{G}$.

The X-ray synchrotron emission from Cas A seems to decline rather rapidly (Patnaude et al. 2011), $1.5-2 \% \mathrm{yr}^{-1}$, which is much faster than the radio flux decline. This can be understood best by taking into account that the synchrotron cut-off energy is declining as a result of the slowing down of the shock. The decline is somewhat fast compared to the expectations based on a simple, Sedov-type shock speed evolution.

The other X-ray bright, young SNR, Tycho, also showed some surprising results. First of all, the ejecta seem to lie very close to the shock front (Warren et al. 2005; CassamChenaï et al. 2007), which can be attributed to a very high compression ratio $\chi>4$ as can be expected according to non-linear DSA theories (e.g. Decourchelle et al. 2001; Vink et al. 2010; Kosenko et al. 2011). However, there is currently a debate whether hydrodynamic instabilities may be another way to explain the appearance of ejecta close to the shock front (Orlando et al. 2012).

Another strange X-ray feature in Tycho's SNR is the appearance of a comb-like X-ray synchrotron structure, which has been interpreted as filamentation with a pattern-size matching the gyro-radius of the highest energy protons, which would then have to be close to $10^{15} \mathrm{eV}$ (Eriksen et al. 2011; Bykov et al. 2011, Fig. 1). However, the coherence of the pattern, its orientation and its singular appearance need further clarification.

\section{The maximum cosmic ray energies in SNRs}

The X-ray synchrotron spectra indicate that magnetic fields in young SNRs can be as high as $B_{2} \sim 500 \mu \mathrm{G}$. The evidence that the magnetic field is amplified in the cosmic ray precursor, and that it scales with $B^{2} \propto \rho_{0} V_{\mathrm{s}}^{\alpha}$, suggests that the highest energies may be reached in the early phase of the SNR evolution. Moreover, it helps if the SNR is evolving in a dense stellar wind. In that case $\rho_{0}=\dot{M} / 4 \pi r^{-2} v_{w}$, which means that densities, and hence magnetic fields, are larger in the earliest phases of the SNR evolution (see Ptuskin 

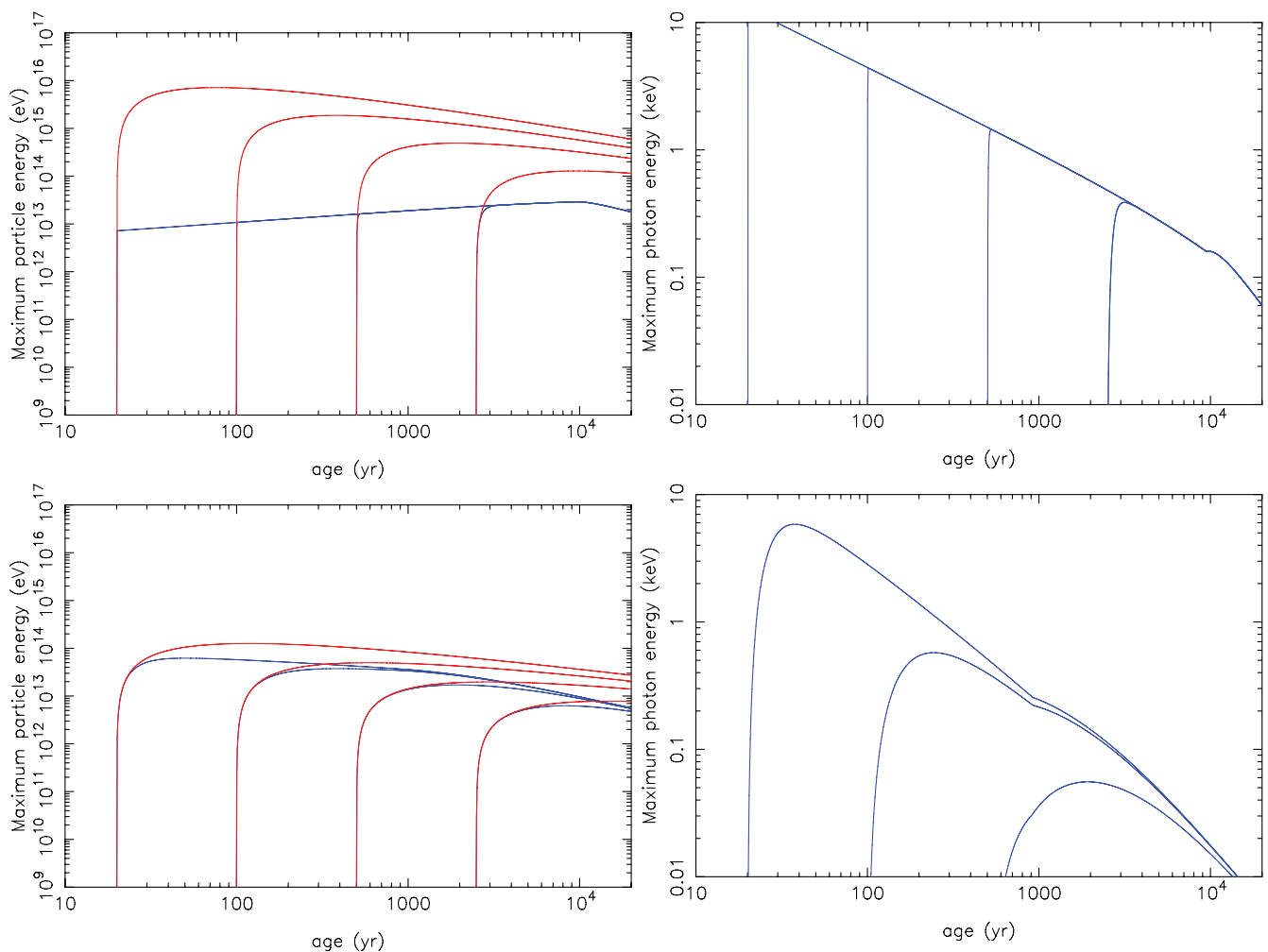

Figure 2. Top left: Maximum proton (top, red lines) and electron (blue) energies for particles that started to be accelerated at selected intervals. The parameters are chosen to match Cas A at the current epoch (at $t=330$ yr: $B_{2}=500 \mu \mathrm{G}, \eta=5, V_{\mathrm{s}}=5200 \mathrm{~km} \mathrm{~s}^{-1}, R_{\mathrm{s}}=2.6 \mathrm{pc}$, $\left.n_{0}=2 \mathrm{~cm}^{-2}, R_{\mathrm{s}} \propto t^{2 / 3}\right)$. The magnetic field scales as $B^{2} \propto \rho_{0} V_{\mathrm{s}}^{3}$, but is held constant once $B_{0}=10 \mu \mathrm{G}$. Adiabatic losses are taken into account, and result in the downward slope of the proton spectra, once the maximum has been reached. Top right: The resulting characteristic maximum synchrotron photon energy. Although the maximum electron keeps going up with time till $t \approx 10^{4} \mathrm{yr}$, the photon energy declines rapidly because the magnetic field is declining. Bottom panels: similar to the top panels, but with a different magnetic field $B_{0}=30 \mu \mathrm{G}$, a flat circumstellar medium, and $R_{\mathrm{s}} \propto t^{0.5}$.

\& Zirakashvili 2005; Schure et al. 2010). Moreover, the high early density results in more particles entering the shock front at early times.

The effects of magnetic field evolution on the maximum electron and proton energies is illustrated in Fig. 2. It shows that for a SNR with characteristics typical for Cas A the maximum energy that a proton that started to be accelerated at a SNR age of $t=20 \mathrm{yr}$ could reach $10^{16} \mathrm{eV}$. The high magnetic field makes that the maximum electron energy for an electron injected at $t=20 \mathrm{yr}$ is only $10^{13} \mathrm{eV}$. Note that the electrons are accelerated almost instantaneously, and are limited by synchrotron energy losses. Although the maximum electron energy goes up with age, the maximum characteristic photon energy declines with age, due to the decline of the magnetic field.

Note that adiabatic losses make that protons start losing energy after reaching the maximum energies. It is, due to the decline in magnetic field their diffusion length scales are rapidly increasing and become $l_{\text {diff }}>0.1 R_{\mathrm{S}}$ shortly after reaching the peak energy. As a result they become detached from the SNRs and escape. The bottom panels of 
Fig. 2 shows similar calculations, but now for a much lower magnetic field, resulting in an electron spectrum that is nearly age limited.

\section{Gamma-ray emission from supernova remnants}

EGRET (Esposito et al. 1996) identified several gamma-ray sources that appeared to be associated with SNRs. However, the big break-through in the field of gamma-ray studies of SNRs came with the coming of age of Cherenkov telescopes such as HEGRA, H.E.S.S., MAGIC, and Veritas, which cover very high energy gamma-rays (VHE gammarays, $\sim 0.1-100 \mathrm{TeV})$. The first detected shell-type SNR to be detected was Cas A by HEGRA(Aharonian et al. 2001), although it was not as bright as perhaps anticipated. A source that was very bright was the otherwise somewhat obscure SNR RX J1713.7-3946 (RXJ173, Aharonian et al. 2004, 2007), which has a VHE spectrum that extends up to $100 \mathrm{TeV}$, indicating primary particles with energies close to $10^{15} \mathrm{eV}$. Since these initial discoveries many more young SNRs have been detected in VHE gamma-rays, including Vela Jr (Aharonian et al. 2005), RCW 86 (Aharonian et al. 2009) and Tycho's SNR (Acciari et al. 2011).

However, what is not immediately clear from the VHE spectra alone is whether the emission is caused by accelerated electrons (leptonic cosmic rays) or from accelerated protons and other atomic nuclei (hadronic cosmic rays). Accelerated electrons cause gamma-ray emission either due to bremsstrahlung, which requires a sufficiently high local plasma density, or due to inverse Compton scattering of cosmic microwave background photons (or other strong photon fields). The mechanism by which accelerated protons cause gamma-ray emission is pion-decay: hadronic cosmic rays colliding with the background atoms result in the production of, among others, neutral pions, which decay emitting two photons.

In particular for RXJ1713 a strong debate ensued over the nature of the gamma-ray emission (Katz \& Waxman 2008; Berezhko \& Völk 2008; Acero et al. 2009; Berezhko \& Völk 2010; Ellison et al. 2010). This debates has now settled somewhat in favor of inverse Compton scattering due to the detection of RXJ1713 by the Fermi-LAT instrument (Abdo \& Fermi LAT Collaboration 2011), which indicates a rather hard spectrum that is more consistent with inverse Compton scattering than with pion decay. Also the detection of several other young SNRs by Fermi shows that in many cases the emission seems to be caused by inverse Compton scattering (e.g. RCW 86 Lemoine-Goumard et al. 2012). A likely exception among the young SNRs seems to be Tycho's SNR (Acciari et al. 2011; Giordano et al. 2012). For Tycho it also appears that the energy in cosmic rays inside the SNR is about $10 \%$ of the explosion energy, whereas for Cas A it seems to be only $4 \%$ (Abdo et al. 2010).

The case for pion-decay emission is more compelling for several mature SNRs. Many of the Fermi and AGILE detected mature SNRs fall in the class of the so-called mixedmorphology SNRs (see ?Helder et al. 2012, for a discusion), which have radio shells that are too cool to emit X-rays, but interiors that are hot enough to emit thermal X-ray emission. These SNRs are likely shaped by their high density environments, slowing down the shock front rapidly, resulting in cool SNR shells. The prevalence of the these SNRs in the Fermi source catalogue already tells us that their gamma-ray emission must be related to their high densities. The high densities also make it likely that the gamma-ray emission is due to either bremsstrahlung or pion-decay. A characteristic of pion-decay gamma-ray spectra is that their is cut-off in the spectrum at low energies, as the energy of the produced photon is $m_{\pi^{0}} / 2=68 \mathrm{MeV}$ in the rest frame of the collision. This 
characteristic low energy cut-off has now been detected by AGILE (Giuliani et al. 2011) and Fermi (Ackermann et al. 2013) for the mixed-morphology SNRs W44 and IC 443.

The Fermi spectra of many mature SNRs show that the spectra are cut-off around $10 \mathrm{GeV}$, indicating that there are not many particles present with energies about a $\mathrm{TeV}$. This suggests that these particles must have escaped the SNR in the past, since in young SNRs higher energy particles are present given the VHE gamma-ray emission. Interestingly, some mature SNRs do have associated $\mathrm{TeV}$ emission, but not immediately from the SNR shell, but coming from nearby molecular clouds. A case in point is the mixed-morphology SNR W28 (Aharonian et al. 2008). This indicates that escape is an important and in the past often neglected aspect of cosmic ray acceleration (see S. Gabici, 2013 , this volume) . It has also to be taken into account when estimating the total energy in cosmic rays produced by a SNR, as some of the cosmic rays may have escaped, perhaps even at an early stage.

\section{Conclusion}

SNRs have for a long time been considered the dominant sources of Galactic cosmic rays. If true SNRs must be able to accelerate particles up to at least $3 \times 10^{15} \mathrm{eV}$ and have an efficiency of around 10\% in transferring energy to accelerated particles.

The rapid development in X-ray and gamma-ray astronomy have helped to strengthen the case for cosmic ray acceleration by SNRs. The presence of X-ray synchrotron emission and the narrowness of the X-ray synchrotron emitting regions indicate that magnetic fields are amplified near young SNRs, which helps to create the right conditions for accelerating protons up to $3 \times 10^{15} \mathrm{eV}$.

The gamma-ray emission from SNRs indicates that particles in young SNRs can be accelerated to very high energies, but for young SNRs it is not always clear whether the emission is caused by electrons or protons. However, for mature SNRs in dense regions there is now clear evidence for the presence of accelerated protons, although for these mature SNRs the highest energy protons seem to have escaped the SNR shells.

\section{References}

Abdo, A. A. \& Fermi LAT Collaboration. 2011, ArXiv e-prints

Abdo, A. A., et al. 2010, ApJ, 710, L92

Acciari, V. A., et al. 2011, ApJ, 730, L20+

Acero, F., Ballet, J., Decourchelle, A., Lemoine-Goumard, M., Ortega, M., Giacani, E., Dubner, G., \& Cassam-Chenaï, G. 2009, AESA, 505, 157

Ackermann, M., et al. 2013, Science, 339, 807

Aharonian, F. Akhperjanian, A., Barrio, J., et al. 2001, A\&A, 370, 112

Aharonian, F., et al. 2005, A\&A, 437, L7

-. 2007, A\&A, 464, 235

-. 2008, $A \& A, 481,401$

-. 2009, ApJ, 692, 1500

Aharonian, F. A. \& Atoyan, A. M. 1999, A\&A, 351, 330

Aharonian, F. A., et al. 2004, Nature, 432, 75

Axford, W. I., Leer, E., \& Skadron, G. 1977, in International Cosmic Ray Conference, Vol. 11, International Cosmic Ray Conference, 132-+

Baade, W. \& Zwicky, F. 1934a, Contributions from the Mount Wilson Observatory, vol. 3, pp.73-78, 3,73

-. 1934b, Physical Review, 46, 76

Ballet, J. 2006, Advances in Space Research, 37, 1902

Bamba, A., Ueno, M., Nakajima, H., \& Koyama, K. 2004, ApJ, 602, 257 
Bell, A. R. 1978, MNRAS,, 182, 147

—. 2004, MNRAS, 353,550

Berezhko, E. G., Ksenofontov, L. T., \& Völk, H. J. 2003, A\&\&A, 412, L11

Berezhko, E. G. \& Völk, H. J. 2008, A\&̊A, 492, 695

一. 2010, A\&A, 511, A34+

Blandford, R. D. \& Ostriker, J. P. 1978, ApJ, 221, L29

Bykov, A. M., Ellison, D. C., Osipov, S. M., Pavlov, G. G., \& Uvarov, Y. A. 2011, ApJ, 735, L40

Carlton, A. K., Borkowski, K. J., Reynolds, S. P., Hwang, U., Petre, R., Green, D. A., Krishnamurthy, K., \& Willett, R. 2011, ArXiv e-prints

Cassam-Chenaï, G., Hughes, J. P., Ballet, J., \& Decourchelle, A. 2007, ApJ, 665, 315

Decourchelle, A., et al. 2001, A\&A, 365, L218

Delaney, T. \& Rudnick, L. 2003, ApJ, 589, 818

Drury, L. O. \& Downes, T. P. 2012, MNRAS, 427, 2308

Ellison, D. C., Patnaude, D. J., Slane, P., \& Raymond, J. 2010, ApJ, 712, 287

Eriksen, K. A., Hughes, J. P., Badenes, C., Fesen, R., Ghavamian, P., Moffett, D., Plucinksy, P. P., Rakowski, C. E., Reynoso, E. M., \& Slane, P. 2011, ApJ, 728, L28

Esposito, J. A., Hunter, S. D., Kanbach, G., \& Sreekumar, P. 1996, ApJ, 461, 820

Ginzburg, V. L. 1959, in IAU Symposium, Vol. 9, URSI Symp. 1: Paris Symposium on Radio Astronomy, ed. R. N. Bracewell, 589

Ginzburg, V. L. \& Syrovatskii, S. I. 1964, The Origin of Cosmic Rays

Giordano, F., Naumann-Godo, M., Ballet, J., Bechtol, K., Funk, S., Lande, J., Mazziotta, M. N., Rainò, S., Tanaka, T., Tibolla, O., \& Uchiyama, Y. 2012, ApJ, 744, L2

Giuliani, A., et al. 2011, ApJ, 742, L30

Helder, E., Vink, J., Bykov, A., Ohira, Y., Raymond, J., \& Terrier, R. 2012, Space Science Rev., 1,1

Helder, E. A. \& Vink, J. 2008, ApJ, 686, 1094

Hinton, J. A. \& Hofmann, W. 2009, ARAA, 47, 523

Katz, B. \& Waxman, E. 2008, Journal of Cosmology and Astro-Particle Physics, 1, 18

Kosenko, D., Blinnikov, S. I., \& Vink, J. 2011, ArXiv e-prints

Koyama, K., et al. 1995, Nature, 378, 255

Krymskii, G. F. 1977, Soviet Physics Doklady, 22, 327

Lagage, P. O. \& Cesarsky, C. J. 1983, A\&A, 125, 249

Lemoine-Goumard, M., Renaud, M., Vink, J., Allen, G. E., Bamba, A., Giordano, F., \& Uchiyama, Y. 2012, A\& A, 545, A28

Orlando, S., Bocchino, F., Miceli, M., Petruk, O., \& Pumo, M. L. 2012, ApJ, 749, 156

Patnaude, D. J., Vink, J., Laming, J. M., \& Fesen, R. A. 2011, ApJ, 729, L28+

Ptuskin, V. S. \& Zirakashvili, V. N. 2005, A\&A, 429, 755

Reville, B. \& Bell, A. R. 2013, MNRAS, 430, 2873

Reynolds, S. P. 2008, ARAA, 46, 89

Schure, K. M., Achterberg, A., Keppens, R., \& Vink, J. 2010, MNRAS,, 406, 2633

Shklovsky, J. S. 1968, Supernovae (Interscience Monographs and Texts in Physics and Astronomy, London: Wiley, 1968)

Uchiyama, Y. \& Aharonian, F. A. 2008, ApJ, 677, L105

Vink, J. 2008, in American Institute of Physics Conference Series, Vol. 1085, American Institute of Physics Conference Series, 169-180

Vink, J. 2012a, ArXiv e-prints

-. 2012b, A\& Ar, 20, 49

Vink, J., Bloemen, H., Kaastra, J. S., \& Bleeker, J. A. M. 1998, A\&A, 339, 201

Vink, J. \& Laming, J. M. 2003, ApJ, 584, 758

Vink, J., Yamazaki, R., Helder, E. A., \& Schure, K. M. 2010, ApJ, 722, 1727

Völk, H. J., Berezhko, E. G., \& Ksenofontov, L. T. 2005, A\&A, 433, 229

Warren, J. S., et al. 2005, ApJ, 634, 376

Webber, W. R. 1998, ApJ, 506, 329

Zirakashvili, V. N. \& Aharonian, F. 2007, A\& A, 465, 695 


\section{Discussion}

Koo: Yesterday, we heard that as much as $90 \%$ of SNe may go off inside superbubbles. I guess the cosmic acceleration in such environment could be different from what we learned for single isolated SNRs. Could you comment on that?

VINK: Yes, if bubble is hot you may have for a longest time low Mach numbers, hampering efficient acceleration. However, Bykov e.g. have suggested that second order Fermi may take place in bubble, using Alfven waves excited by many SNRs together. 2nd order Fermi is slow, but the bubbles have longer lifetimes than SNRs. 